\title{
Development of Nanosatellite based Image Retrieval System
}

\author{
Jean Marie Gashayija \\ Cape Peninsula University of Technology \\ Bellville campus
}

\author{
Almarie Bierman \\ Cape Peninsula University of Technology \\ Bellville Campus
}

\begin{abstract}
Rapid development of nanosatellites has led to many unique and innovative space applications. These tiny satellites have come a long way since Sputnik, the first satellite that was launched in 1957, weighing $83 \mathrm{~kg}$. The success of the CubeSat has revolutionized space technology. Typically, these CubeSat fall into categories of nanosatellites weighing no more than $1.33 \mathrm{Kg}$ and using less power than a five watt light bulb. Mostly, CubeSat has one or more payloads such as Imaging Payload, Scientific payload and High Frequency (HF) Radio Beacon.

With the Imaging payload their task is to capture low or high resolution images of the earth observation missions. With the steadily increasing demand for CubeSat imaging payload missions, several nano-satellites have been launched, and thousands of low/high resolution images are acquired every day and transmitted to ground stations. This leads to an exponential increase in the number of low/high resolution images in databases. Therefore, how to retrieve useful images quickly and accurately from a huge and unstructured image database becomes a challenge.
\end{abstract}

In this project, it proposes the use of a content-based image retrieval (CBIR) system relying on a combination of the three low level features such as color, shape and texture features. In order to accurately classify and retrieve useful information in a huge image database. The evaluation performance of the proposed system when compared to other existing systems provides a precision value of 1 or $100 \%$. It can be concluded that the proposed system is able to classify images and retrieve results in a reasonable time of at least 15 seconds.

\section{General Terms}

Image Retrieval, Content Based Image Retrieval and Pattern Recognition

\section{Keywords}

CubeSat, Content Based Retrieval System, Color Feature, Shape Feature, Texture Features and Image Database.

\section{INTRODUCTION}

A CubeSat is a Cube $10 \mathrm{~cm} \times 10 \mathrm{~cm} \times 10 \mathrm{~cm}$. These tiny satellites have come a long way since Sputnik, the first satellite that was launched in 1957, weighing $83 \mathrm{~kg}$. The success of the CubeSat has revolutionized space technology. Typically, these CubeSat fall into categories and use less power than a five watt light bulb. Mostly, CubeSat has one or more payloads such as Imaging Payload, Scientific payload and $\mathrm{HF}$ radio Beacon. With the Imaging payload their task is to capture low or high resolution images of the earth observation missions [8].

As stated in [13], the main objective of their (ICUBE1), which was the first Pakistan CubeSat satellite, was to allow students to familiarize themselves with satellite imaging system which involves image capture, image analysis, compression, storage and retrieval. However, their objectives were not to get high resolution earth images, but to take low resolution images, store them and successfully send them to a ground station. Other CubeSat imaging payloads are such of Masat-1 satellite which is first Hungarian Satellite developed and built by students at the Technical University of Budapest, providing telemetric data as well as VGA resolution images [18].

As stated in [14], their ESTCube-1 Satellite is a low-power imaging system having a camera module weighing $30 \mathrm{~g}$ with onboard image processing. The imaging system is optimized for use in a single unit CubeSat. The camera has $4.4 \mathrm{~mm}$ telecentric lens, a 10bit $640 \times 480$ pixel CMOS image sensor. The resolution of the assembled system is $12.7 \mathrm{~mm}$ and $1 \mathrm{~km}$ per pixel at distances of $10 \mathrm{~m}$ and $700 \mathrm{~km}$ respectively.

With the steadily expanding demand for remotely sensed images, many satellites have been launched, and thousands of images are acquired every day. This leads to an exponential increase in the quantity of remotely sensed images in databases. Therefore, how to retrieve useful images quickly and accurately from a huge and unstructured image database becomes a challenge [17].

As stated in [10], the term Content Based Image Retrieval System (CBIR) is defined as a process to retrieve a stored image from a database by supplying an image as query instead of text, using the image's extracted low level features such as color, texture and shape descriptors. The purpose of the CBIR system is to retrieve an image that is most similar to a queried image within the image database. The most important challenge of the CBIR system is to determine the exact/appropriate matching image of a database to the query image. In this project, it is proposed to classify satellite images using the CBIR system to associate a query image to similar group/class of stored images. As stated in [3], the CBIR system, it's usual to group the image features into three classes such as color, shape and texture.

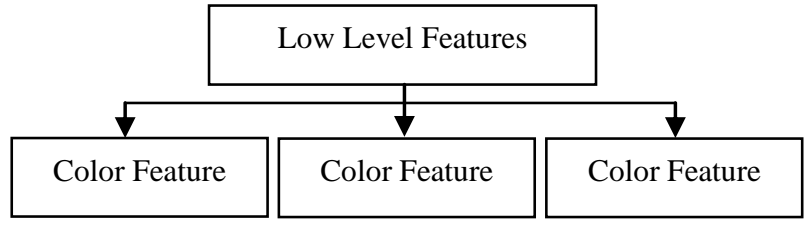

Figure 1: Categories of the low level features

As shown in the Figure 1, there are broadly three categories of low level features such as color, shape and texture. Color features are by far the most common visual feature used in CBIR system, primarily because of the simplicity of extracting color information from images. CBIR systems mostly use color histograms [32], [29], [39], color moments [30], color correlograms [24], color coherence vectors and dominant color descriptors to represent color.

Color histogram is a standard demonstration of color characteristic in CBIR systems. It is very efficient in the description of both local and global features of colors. It 
computes the chromatic information and invariant of image along the viewed axes for translation and rotation. Color histogram is a fundamental technique for retrieving images and extensively used in CBIR systems [41].

As stated in [37], color histograms also have some limitations: they provide no spatial information but merely describe which colors are present in the image and in what quantities. They are also sensitive to both compression artifacts and changes in overall image brightness. When the large scale image database computes a histogram, its efficiency is not satisfactory. To overcome this conflict, joint histogram technique is introduced.

Shape feature is an important low level image feature being used in CBIR system. Generally, there are two categories of shape feature, such as contour-based and region- based. The contour-based uses only the contour of the object's shape while the region- based methods use the internal details in addition to the contour [22]. Examples of the contour-based methods are chain code [35], shape signature [28], polygonal and Fourier Descriptors [7], Wavelet Descriptors and approximation [42]. Examples of the region-based methods are Mathematical Morphology [31], [12], [27], geometric moments [26], Zernike moments [33],[15], grid representation [6] and area which exploit only shape interior information [43].

Texture features are also an important visual feature which refers to innate surface properties of an object and their relationship to the surrounding environment. Many objects in an image can be distinguished solely by their textures without any other information. There is no universal definition of texture. Texture may consist of some basic primitives and may also describe the structural arrangement of a region and the relationship to the surrounding regions [40], [38].

In conventional texture features used for CBIR, there is a gray level co-occurrence matrix [25], [20], [11], Wavelet transforms [21], Local Binary Pattern [5], Tamura's texture [9], Gabor's Filters [9], Markov Random Field [36], [34].

\section{METHODOLOG OF PROPOSED}

\section{DESIGN}

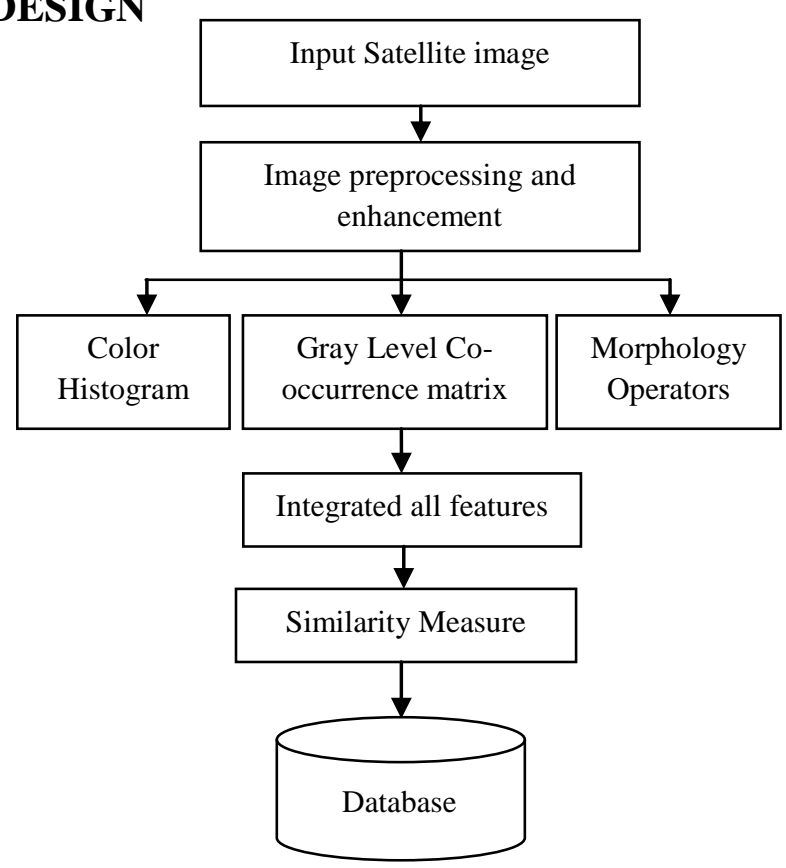

Figure 2: Block Diagram of Proposed Design
As illustrated in the Figure 2, a block diagram of the proposed work is given. It shows all building blocks of the system design. Therefore, the processing steps used in this project are as follows:

\subsection{Image pre-processing and enhancement}

Digital Satellite images are frequently distorted by noise due to errors resulting from noisy sensor and communication channels. This error that arises adjusts the intensity of a number of pixels while some remain unchanged [2]. In order to eliminate image noise and improve the affected image quality in this project, the proposed image pre-processing technique to be used to eliminate noise and improve quality of image is median filter. This filter eliminates the noise in the image while preserving the image boundary information and improves the image quality as well. Typically, a median filter considers each pixel in the image and looks at its nearby neighbours to decide whether it is a representative of its surroundings.

Instead of simply replacing the pixel value with the mean of neighboring pixel values, it changes it with the median of individual values. The median is determined by sorting all the pixel values from the surrounding neighbourhood into numerical order and then replacing the pixel being considered with the central pixel value. In median filtering, the neighboring pixels are ranked according to brightness (Intensity) and the median value becomes the new value for the central pixel [4].

\subsection{Color Feature}

As shown in Figure 3, for the proposed task of color feature, the color histogram algorithm was chosen. It is very effective, simple and easy to implement. The use of a color histogram is the most common way for representing color feature. A histogram counts the number of pixels of each kind and can be rapidly created by reading each image pixel just once and incrementing the appropriate bin of histogram. It is relatively invariant to translation and rotation. As stated in [41], the color histogram was compared to other color features in order to retrieve images. They found when all large number of bins of histogram are used it leads to a decline in the performance of the system. Therefore, in the proposed design all the image's histogram bins will be normalized and reduced to 16 bins for better retrieval rate.

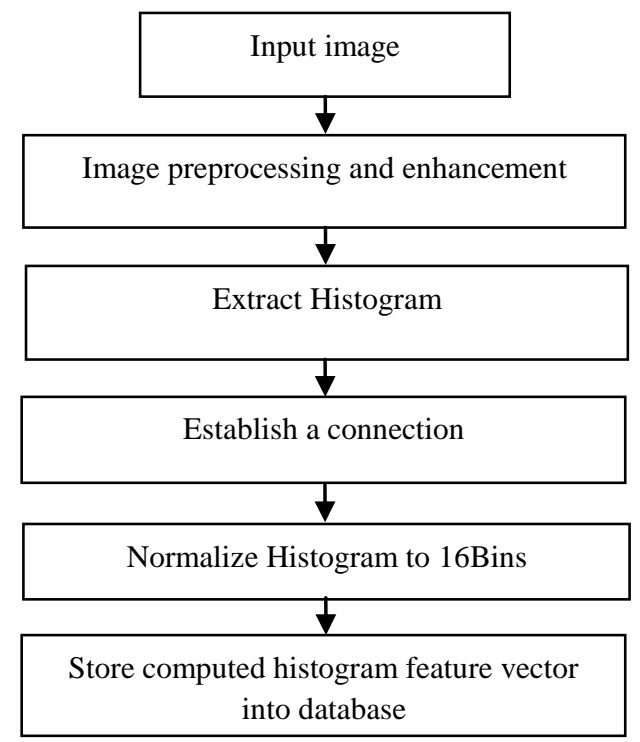

Figure 3: Block Diagram of proposed Color Histogram method 


\subsection{Texture Feature}

As shown in the Figure 4, the proposed method for texture feature is Grey Level Co-Occurrence matrix texture (GLCM). The GLCM is one of the widely used methods to perform textural feature measurements. The GLCM varieties of measures are used to extract useful textural information from co-occurrence matrix. For this project, in order to extract useful textural information, the selected GLCM measurements texture features were Contrast, Correlation, Energy and Homogeneity.

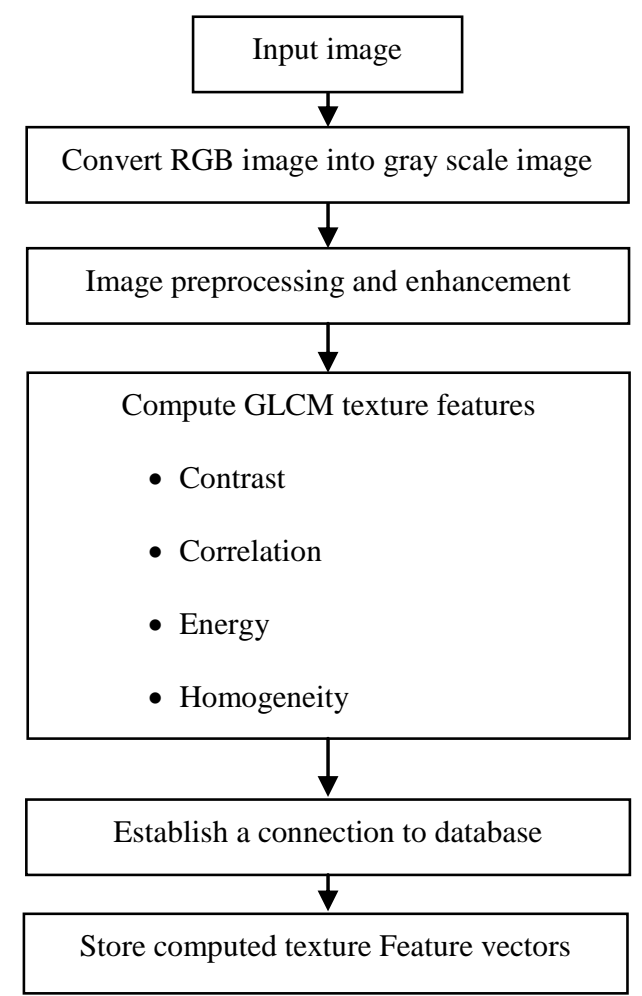

Figure 4: Block diagram of the proposed GLCM texture Feature

\subsection{Shape Feature}

As shown in the Figure 5, the proposed method for shape feature is mathematical morphology. Mathematical morphology is a tool for extracting image components that are useful for representation and description. Generally, morphological operations are based on a simple expanding and shrinking operation using binary, gray level and color images. The basic morphological set transformations are an erosion and dilation operation which involves the interaction between the image and structuring elements of a desired shape (i.e., a circular disk and a square).

In this project, the image and structuring elements (SE) set need not be restricted to set in the $2 \mathrm{D}$ plane only, as SE normally can be of one, two, three or more dimensions. The erosion and dilation can be used in series and parallel to give other transformations including thickening, thinning, skeletonisation and many others. Therefore, dilation expands an image object and erosion shrinks it. The use of these basic operations gives rise to opening and closing transformations. Opening generally smooths a contour in an image, breaking narrow isthmuses and eliminating thin protrusions. Closing tends to narrow smooth sections of contours, fusing narrow breaks and long thin gulfs, eliminating small holes and filling gaps in contours.

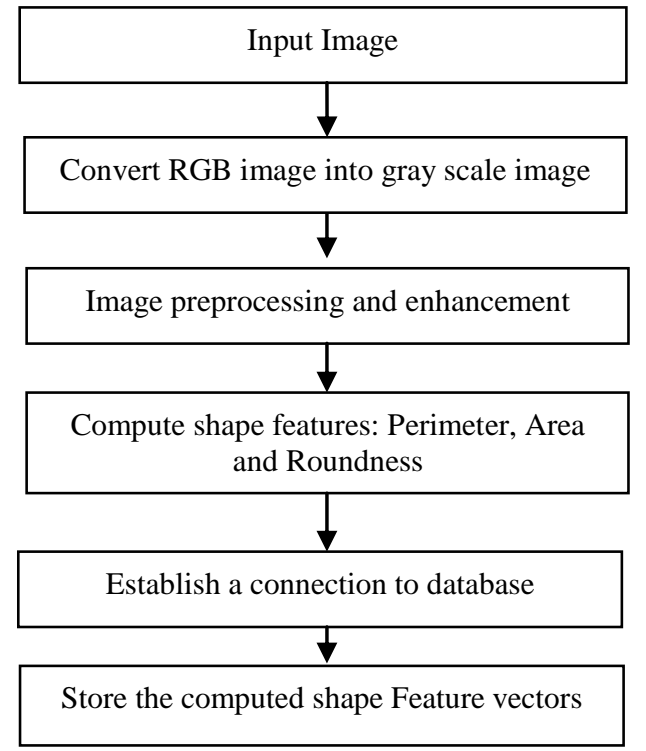

Figure 5: Block diagram of mathematical morphology shape feature

\subsection{Similarity Measure}

After computing the feature vectors of query image, it will be compared to the feature vectors of the database's images which were processed initially and stored. Therefore, when comparison is done, a distance measure value is ranked according to the lowest to highest value according to image and their feature vector compared against the query image's feature. The similarity retrieval results range from smaller to high values. The smaller the distance value, the greater chance that query image is similar to those in the database. As stated in [40], the similarity between the query images against images in the database can be computed using similarity Euclidean distance measure described in their proposed work.

\subsection{Database of Images}

The database consists of a number of satellite high resolution images such as Masat-1 Satellite, Ikonos and as well as Quick Birds satellite. Each satellite image's content is extracted using shape feature method; color feature method and texture feature method previously discussed in section 2.2 up to section 2.4 and all feature vectors are stored in the database (MySQL 5.5 version). A variety of satellite high resolution images (such as Ikonos, Quick-Bird and Masat-1) of different sizes were downloaded, extracted and their feature vectors populated in the database to form an image collection for the experimental purpose.

\section{EXPERIMENTAL SET-UP}

As shown in the Figure 6, the project was development using Matlab 2009b as a software platform on a personal computer running a Windows 7 operating system with i5-520M Processor, 3.33 GHZ processing speed and $4 \mathrm{G}$ of memory (RAM) and 500 of hard Disk Drive and MySQL Database. The matlab platform has toolboxes used to prototype graphical user interface which allows users to interact. It also has in-built image processing functions which are valuable in extracting features vectors in an image. The database used is a MySQL Database 5.5 version. It is an open source database, simple to store and retrieve data in it and easy to interface with the matlab platform. However, communication between Matlab-MySQL databases was achieved by using ODBC 
Microsoft and a MySQL driver equivalent to the version of both Matlab as well MySQL.

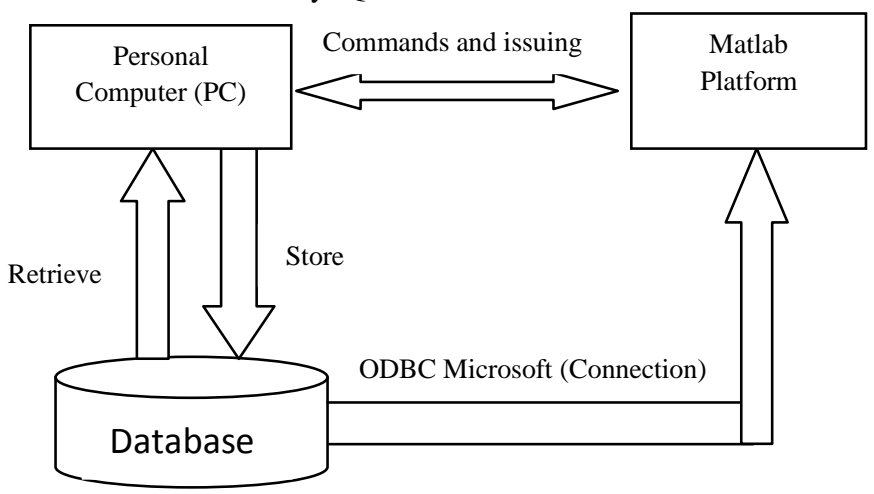

Figure 6: Experimental set-up

\section{EXPERIMENTAL RESULTS}

As shown in Figure 7, a combined graphical user interface with proposed color histogram, Gray level co-occurrence Matrix (GLCM) and Mathematical Morphology algorithm methods were tested. The query image of Masat-1 high resolution images were used to retrieve similarity results in the database. The retrieval time was 6.64438 seconds.

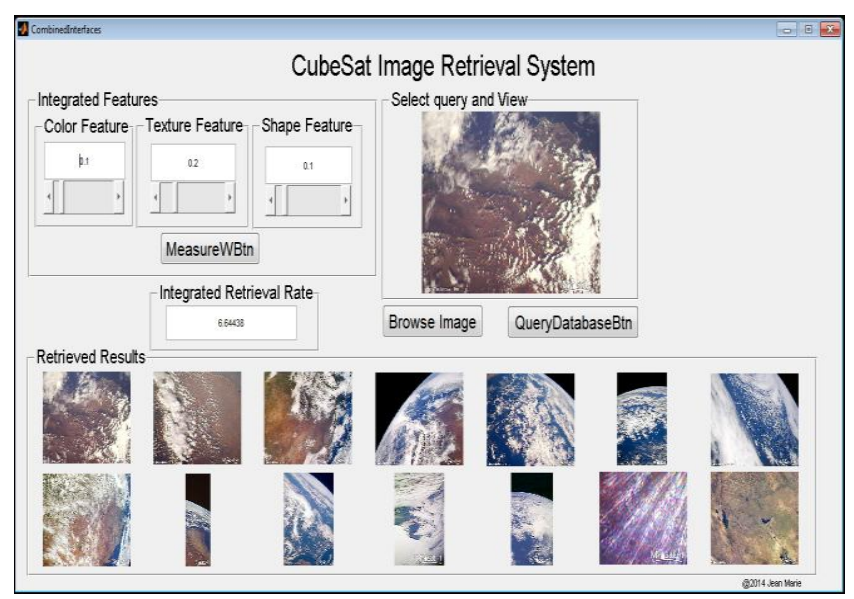

Figure 7: Retrieved Results of Masat-1 Images

In Figure 8, a second testing on a built graphical user interface with combined features was demonstrated on large satellite with high resolution images such as Ikonos and Quick-Bird satellites. However, due to their large size their retrieval rate was 40.0602 seconds compared to nanosatellite (CubeSat) retrieval rate of 6.64438 seconds. Therefore, it can be said that this built system not only can be used for nanosatellites but also for large satellite systems to classify and retrieve images in the large database.

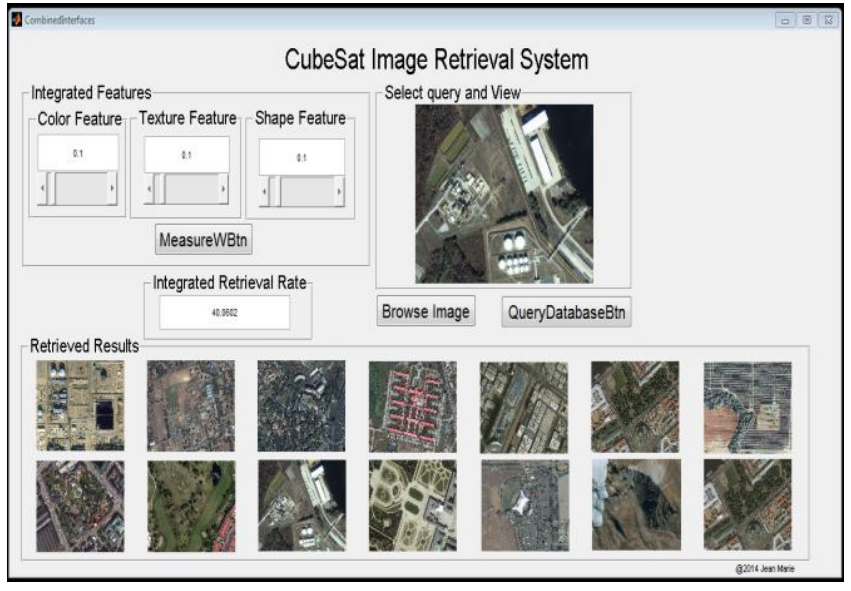

Figure 8: Retrieved results of Ikonos and Quick bird High resolution images

As shown in Figure 9, a Wang database (having ten groups) was used to compare the proposed system against other existing systems. In the figure buses group was evaluated together with other groups in order to see if the developed system performance gives accurate results in a reasonable retrieval time of 6.12791 seconds.

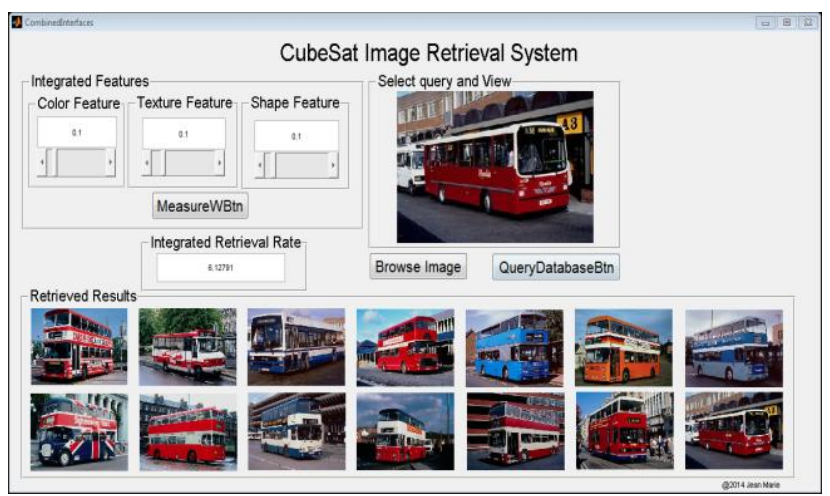

Figure 9: Retrieved results of Wang Database images

In the Figure 10, each algorithm retrieval time was plotted against each class. It shows that GLCM algorithm tend to be a bit slow compared to other algorithms. But once an integrated algorithm is achieved the system behaves much better compared to its individual algorithm.

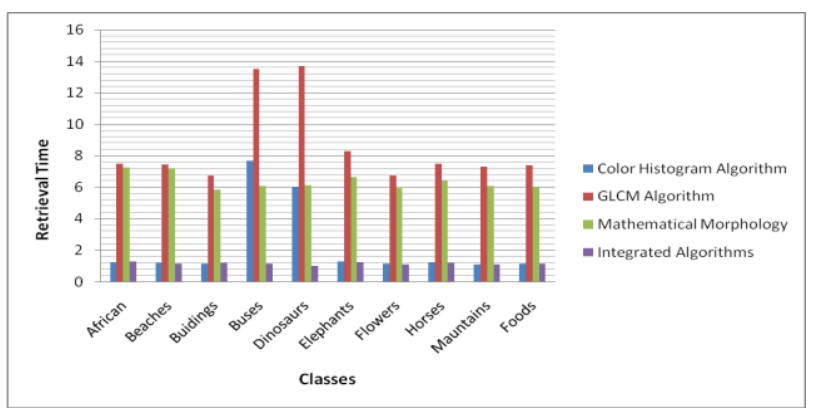

Figure 10: Algorithms Efficiency(Retrieval time)

\section{PERFORMANCE EVALUATION}

In CBIR system, the main concern is performance efficiency and retrieval effectiveness. The standard measures of evaluating the performance of a CBIR system are precision and recall. These methods are used to evaluate the 
performance of the system. Precision is defined as the ratio of the number of the relevant images retrieved to the number of images retrieved. It is a measure of retrieval based on equation 1, whereas recall is defined as the ratio of the number of retrieved relevant images to the total number of relevant images in the database. Recall is a measure of the robustness of the retrieval. It is computed using equation 2 .

\section{Precision $=\frac{\text { Number of relevant images retrieved }}{\text { Total number of images retrieved }}$}

(1)

Recall $=\frac{\text { Number of relevant images retrieved }}{\text { Total number of images in the database }}$

(2)

Note that, due to the lack of standard image database, to compare the proposed system retrieved results with other existing system's results, they also have to use the same database. Therefore most existing systems relied on Wang database of 1000 images of ten groups, each comprising 100 images. As shown in Table 1, the comparisons of the proposed system with other existing systems are those of [16], [23], [1] and [19].

Table 1: Comparison of precision of the proposed system against others systems

\begin{tabular}{|l|r|r|r|r|r|}
\hline Classes & $\begin{array}{l}\text { L16] } \\
\text { Lin et } \\
\text { al.,2009 }\end{array}$ & $\begin{array}{l}\text { [23] } \\
\text { Raghupathi } \\
\text { et al.,2010 }\end{array}$ & $\begin{array}{l}\text { [1] Afifi's } \\
\text { System(2011) }\end{array}$ & $\begin{array}{l}\text { [19] } \\
\text { Mikharq's } \\
\text { System(2013) }\end{array}$ & $\begin{array}{l}\text { Proposed } \\
\text { System }\end{array}$ \\
\hline $\begin{array}{l}\text { Africa } \\
\text { people }\end{array}$ & 0.68 & 0.75 & 0.71 & 1 & 1 \\
\hline Beaches & 0.62 & 0.6 & 0.86 & 0.93 & 1 \\
\hline Buildings & 0.71 & 0.43 & 0.83 & 0.61 & 1 \\
\hline Buses & 0.92 & 0.69 & 0.86 & 1 & 1 \\
\hline Dinosaurs & 0.97 & 1 & 1 & 1 & 1 \\
\hline Elephants & 0.86 & 0.72 & 0.71 & 0.63 & 1 \\
\hline Flowers & 0.76 & 0.93 & 0.94 & 1 & 1 \\
\hline Horses & 0.87 & 0.91 & 0.57 & 1 & 1 \\
\hline Mauntains & 0.49 & 0.36 & 0.43 & 0.65 & 1 \\
\hline Foods & 0.77 & 0.65 & 0.98 & 1 & 1 \\
\hline Total & 0.76 & 0.7 & 0.79 & 0.88 & 1 \\
\hline
\end{tabular}

In the Figure 11, is a comparison of precision of the proposed system against existing systems. It's based on each class precision value shown previously in Table 1 . Therefore, it can be concluded that the proposed system outperforms the other system in most of the classes and offers accurate results.

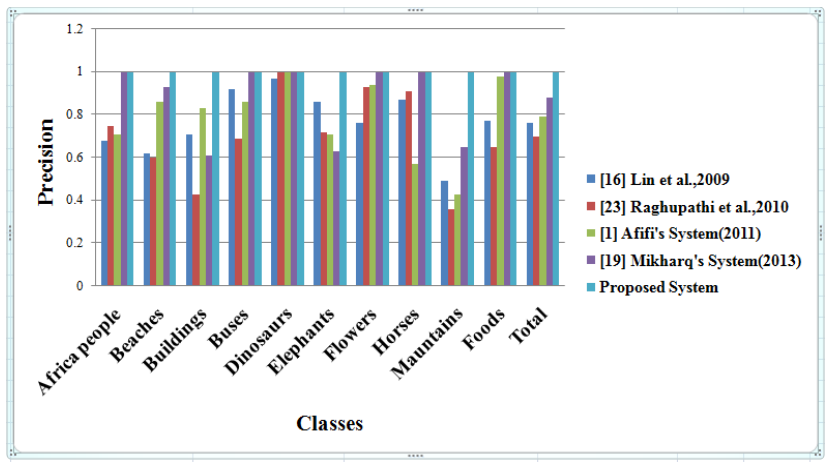

Figure 11: Comparison of Precision of the proposed system against existing systems

\section{DISCUSSION}

In conclusion, the main purpose of developing of the nanosatellite based retrieval system was achieved. The proposed system relies on three low level features such as color, shape and texture in order to classify and provide accurate retrieval results. When the proposed system is compared to other existing systems, it outperforms them with precision of 1 or $100 \%$. In this proposed system, images were not reduced and retrieval time remained approximately seven seconds on nanosatellite images and as well on Wang database images. However, when this system needs to be used for a large satellite database, the author suggests that the size of image needs to be reduced or cropped to minimize the retrieval rate.

\section{ACKNOWLEDGMENTS}

Thanks are due to my supervisor Prof.Bierman for guidance in times where I was in doubts, without her this journal could not have been a written success. Thanks also due Roger Grahams for grammar checking of this work.

\section{REFERENCES}

[1] Afifi, A. J. 2011. Image retrieval based on content using color feature. M.Tech Thesis, Islamic University of Gaza.

[2] Asubam, W. B. 2011. Improved median filtering algorithm for the reduction of impulse noise in corrupted 2D greyscales images. MSc Thesis, Kwame Nkrumah University of Science and Technology.

[3] Chaudhari, R., \& Patil, A. M. 2012. Content based image retrieval using color and shape features. International Journal of Advanced using color and shape features,1(5):386-392,November.

[4] Chun-Yu, N., Shu-Fen, L. \& Ming, Q. 2009. Research on removing noise in medical image based on median filter method. Proceedings of the 2009 IEEE symposium on Information Technology(IT) in medicine and Education ,Ji'nan,China,14-16 August 2009.

[5] Das, N., Pal, A., Mazumder, S., Gangopadhyay, D. \& Nasipuri, M. 2013. An SVM based skin disease identification using local binary patterns. The 2013 third International Conference on Advances on Computing and Communications,Kochi,Kerala,India, 29-31 August 2013.

[6] Duan, G., Suzuki, Y. \& Kawagoe, K. 2006. Grid Representation for Efficient similarity search in time series databases. Proceedings of the 2006 22nd International Conference on Data Engineering Workshops(ICDEW'06),Atlanta,Georgia,3-7 April 2006.

[7] El-Ghazal, A., Basir, O. \& Belkasim, S. 2009. Farthest point distance: A new shape signature for fourier descriptors. Signal Proceeding Image Communication,24:572-586.

[8] French South Africa Institute of Technology (FSATI). 2014. http://www.cput.ac.za/fsati/[10 June 2014].

[9] Gebril, M. M. 2011. Structural indexing of satellites images. PhD Thesis, North Carolina A \& T State University.

[10] Haldar, P. \& Mukherjee, J. 2012. Content based image retrieval using histogram,color and edge. International Journal of Computer Applications , 48 (11):25-31. 
[11] Jafarpour, S., Sedghi, Z. \& Amirani, M. C. 2012. A robust brain MRI Classification with GLCM features. International Journal of Computer Applications , 37 (12):1-5,January.

[12] Kalapala, M. 2014. Estimation of tree count from satellite imagery through mathematical morphology. International Journal of Advanced Research in Computer Science and Software Engineering,4(1): 490-495,January.

[13] Khurshid, K., Mahmood, R. \& Islam, Q. U. 2013. A survey of camera modules for CubeSats: Design of imaging payload of ICUBE-1. Proceedings of the 6th International Conference on Recent Advances in Space Technologies,Istanbul,Turkiye, 12-14 June 2013.

[14] Kuuste, H., Eenmae, T., Allik, V., Agu, A., Vendt, R., Ansko, I., Laizans, K., Sunter,I., Latt, S. \& Noorma, M. 2014. Imaging system for nanosatellite proximity operations. Proceedings of the Estonian Academy of Sciences, 2014,63,2s,250-257. Available http://www.kirj.ee/public/proceedings_pdf/2014/issue_2 S/Proc-2014-2S-250-257.pdf.

[15] Li, B., Li, G., Wang, Y., Wang, X., \& Wang, W. 2007. A classification mehod of pear shape based on zernike moment. Proceedings of he 2007 International Conference on Wavelet Analysis and Pattern Recognition,Beijing,China,2-5 November 2007.

[16] Lin, C. H., Chen, R. T., \& Chan, Y. K. 2009. A smart content based image retrieval sysem based on color and texture feature. Image and vision computing , 27, 658665.

[17] Liu, T., Zhang, L., Li, P., \& Lin, H. 2012. Remotely sensed image retrieval based on region level semantic mining. EURASIP Journal on Image and Video Processing , 1 (4): 1-11.

[18] Masat-1. $2014 . \quad$ Projects. http://cubesat.bme.hu/en/projektek/masat-1/[10 June 2014].

[19] Mikhraq, A. K. 2013. Content based image retrieval(CBIR) system based on the clustering and genetic algorithm. MSc. Thesis, Islamic University of Gaza.

[20] Mohanaiah, P., Sathyanarayana, P., \& Gurukumar, L. 2013. Image texture feature extraction using GLCM approach. International Journal of Scientific and Research Publications ,3(5):1-5, May.

[21] Murala, S., Gonde, A. B., \& Maheshwari, R. P. 2009. Color and texture features for image indexing and retrieval. The 2009 IEEE International Advance Computing Conference(IACC2009), Patiala,India, 6-7 March 2009.

[22] Nunes, J. F., Moreira, P. M., \& Tavares, J. S. 2010. Shape based image retrieval and classification. The proceeding of the 2010 5th Iberian Conference on Information System and Technologies(CISTI-2010), Hotel Congreso Santiago de Compostela,Spain, 16-19 June 2010.

[23] Raghupathi, G., Anand, R. S. \& Dewal, M. L. 2010. Color and textures features for content based image retrieval. Proceedings of he second International Conference on multimedia and content based image retreval,21-23 July 2010.
[24] Rautiainen, M. \& Doermann, D. 2002. Temporal color correlograms for video retrieval. Proceedings of the 16th International Conference on Pattern Recognition,Vol2, Qubec City(QC), Canada,11-15 August 2002.

[25] Regniers, O., Da Costa, J. P., Grenier, G., Germain, C. \& Bombrun, L. 2013. Texture based image retrieval and classification of very high resolution maritime pine forest images. IEEE International Geoscience and Remote Sensing Symposium,Melbourne,Australia.

[26] Rizon, M., Yazid, H., Saad, P., Shakaff, A. M., Saad, A. R., Mamat, M. R.,Yaacob,S.,Desa,H. \& Karthigayan,M. 2006. Object detection using geometric invariant moment. America Journal of Applied Sciences , 2 (6):1876-1878.

[27] Roslan, R., Jamil, N. \& Mahmud, R. 2011. Skull stripping magnetic resonance images brain images: Region versus mathematical morphology. International Journal of Computer Information Systems and Industrial Management Applications , 3:150-158.

[28] Safar, M., Shahabi, C., \& Sun, X. 2000. Image rerieval by shape: A comparative study. Institute of Electrical and Electronic Engineers (IEEE), 141-144.

[29] Sergyan, S. 2008. Color histogram features based image classification in content based image retrieval systems. Proceedings of the 2008 6th International Symposium of Applied Machine Intelligence and Informatics(SAMI2008),Herlany,Slovakia, 21-22 January 2008.

[30] Shih, J. L. \& Chen, L. H. 2002. Color image retrieval based on primitives of color moments. Vision,Image and Signal Processing, IEEE Proceedings , 149(6):370-376.

[31] Sivappriya, T., \& Muthukumaran, K. 2014. Cancer cell detection using mathematical Morphology. International Journal of Innovative Research in Compuer and Communication Engineering , 2 (1):3717-3725,March.

[32] Suhasini, P. S., Krishna, K. R. \& Krishna, I. M. 2009. CBIR using color histogram processing. Journal of Theoretical and Applied Information Technology , 6 (1): $116-122$.

[33] Tahmasbi, A., Saki, F., Aghapanah, H. \& Shokouhi, S. B. 2011. A novel breast mass diagnosis system based on zernike moments as shape and density descriptors. The Proceedings of 18th Iranian Conference on BioMedical Engineering,Tehran,Iran,14-16 December 2011.

[34] Tarabalka, Y., Fauvel, M., Chanussot, J., \& Benediktsson, J. A. 2010. SVM and MRF based method for accurate classification of hyperspectral images. IEEE Geoscience and remote sensing letters , 7 (4): 736740, October.

[35] Vaddi, R. S., Boggavarapu, L. P., Vankayalapati, H. D. \& Anne, K. R. 2011. Contour detection using freeman chain code and approximation methods for the real time object detection. Asian Journal of Computer Science and Information Technology(AJCSIT),1(1):15-17.

[36] Valero-Lara, P. 2012. MRF Satellite image classification on GPU. The Proceedings of the $201241^{\text {st }}$ International Conference on Parallel Processing Workshops(ICPP2012),Pittsburg, PA,USA, 10-13 September 2012. 
[37] Valova, I., Rachev, B., \& Vassilakopoulos, M. 2006. Optimization of the algorithm for image retrieval by color features. International Conference on Computer Systems and Technologies-CompSysTech , 1-4.

[38] Vansteenkiste, E., Schoutteet, A., Gautama, S., \& Phillips, W. 2004. Comparing color and textural information in very high resolution satellite image classification. The Proceeding of the 2004 International Conference on Image Processing(ICIP-2004), Singapore, 24-27 October 2004

[39] Wang, S. L., \& Liew, W. C. 2007. Information based color feature representation for image classification. The Proceedings of the $200714^{\text {th }}$ IEEE International Conference on Image Processing(ICIP 2007),San Antonio,Texas, USA,16-19 Sepetember 2007.
[40] Wang, X. Y., Yu, Y. J. \& Yang, H. Y. 2011. An effective image retrieval scheme using color,texture and shape features. Computer Standards and Interfaces ,33 :5968,March

[41] Yasmin, M., Sharif, M. \& Mohsin, S. 2013. Use of low level features for content based image retrieval: Survey. Research Journal of Recent Sciences , 2(11): 65-75,June 2 .

[42] Zhang, D. \& Lu, G. 2004. Review of shape representation and description techniques. Pattern Recognition, 37:1-19.

[43] Zhang, D., \& Lu, G. 2001. Content based shape retrieval using different shape descriptors: A comparative study. The 2001 IEEE International Conference on Multimedia and Expo(IICME), Tokyo, Japan, 22-25 August 2001. 